\title{
Improving the Efficiency of Background Subtraction using Super pixel Extraction and Midpoint for Centroid
}

\author{
K.Suganya Devi, \\ AP/CSE \\ Anna University of Technology \\ Thiruchirappalli(Panruti) \\ Panruti, Tamilnadu, India
}

\author{
N.Malmurugan \\ The Director (Academics) \\ Mahindra Group of Institutions \\ Thirichengode, Tamilnadu, India
}

\author{
S.Poornima \\ Senior Lecturer/CSE \\ Aksheyaa College of Engineering \\ Kanchipuram, Tamilnadu, India
}

\begin{abstract}
This paper deals with an efficient background subtraction of image/frames of video by improving the execution speed, accuracy and reduce the usage of memory. Three important techniques are applied to improve the efficiency: superpixel extraction, canny edge detection and fuzzy c means. On applying the above three methods sequentially, the background of image/video can be segmented from foreground object accurately. The first method reduces the processing data more than $75 \%$. Canny edge detection is an optimized method to detect edges. Fuzzy c means works well and good to segment the overlapped objects in an image/video.
\end{abstract}

\section{General Terms}

Canny edge detection, Feature extraction, Fuzzy c means, Gradient, Segmentation, Smoothing.

\section{Keywords}

Centroid, Edge pixel, Superpixel, Subpixel, Suppression, Thresholding.

\section{INTRODUCTION}

Background subtraction is the process of identifying moving objects from the portion of a video frame that differs significantly from a background scene [4]. The most commonly faced problem in segmenting the foreground object from background scene of a frame and identifying the motion changes of every frame by comparing it with previous frame is the foreground object may move continuously when background scene may be static whereas in some video both the foreground and background objects may move.

This nature of a video leads to produce noise, undetected edges, loss of smoothness, improper segmentation of foreground object especially in overlapped objects and maintaining robustness due to changes in illumination is difficult in the implementation of background subtraction algorithms[5]. Also some more additional work to be done for object tracking, motion analysis and behavior analysis for segmenting the foreground objects. The main aim to segment the foreground object from background scene is, to reduce the amount of data to be processed during comparison of frames. Thus an efficient background subtraction algorithm must have good foreground detection rate, processing rate and adapt quickly to the changes occurring at the background. This paper gives a solution for the above mentioned problems by sequentially applying the following three methods thereby improving the efficiency: (1) extraction of superpixel to reduce the storage capacity, (2) canny edge detection algorithm to detect the edges of objects in the video, and (3) finally fuzzy c means algorithm to segment the foreground object from background scene accurately. Implementation of the above mentioned techniques will be very much useful for many real time applications such as medical diagnosis, human-computer interaction, video surveillance and traffic monitoring.

\subsection{Our Contributions}

our contribution in this paper is, we have suggested a new algorithm for extracting superpixel from the image/frame that is how to group a collection of similar pixels into a superpixel and store the image/frame as superpixels but not individual pixels which reduces the data to be maintained in the memory. Secondly, we have given the idea how to undergo for edge detection using the superpixels. Thirdly, we introduced a new method for identifying the centroid of clusters in fuzzy $c$ means by calculating the midpoint using the edges of the object which plays a major role in improving the accuracy of segmentation for background subtraction.

\subsection{Related Work}

There are a lot of issues faced by many researchers to obtain an efficient background subtraction. Some of the major issues are effect due to noise, loss of smoothness, maintain robustness and improper segmentation of foreground object. Cheng, Gong and Dale [19] listed the related works under background subtraction with the issues in every method. They recovered most of these issues by introducing a series of discriminative learning algorithms namely ILK, SILK and SILK-GPU by comparing their results with other works, But the buffer size used by them is limited in order to maintain constant speed. the proposed ideas of this paper segments the foreground object better than the SILK algorithm.

\section{SUPERPIXEL EXTRACTION}

Superpixel is a polygonal part of a digital image/frame which is larger than normal pixel that is rendered in the similar properties such as color, brightness, texture, vector etc[1]. Superpixels are group of pixels with approximately same pixel values. The main goal of extracting the superpixels from an image/frame is to reduce the size of data that undergoes for background subtraction. Other than this the advantages of using superpixels are computational efficiency, reduction in number of primitives and hypothesis, and less error rate [2] [3].

The important point to be noted during superpixel extraction is pixel collisions must not occur such that none of the pixel must belong to more than one superpixel. Consider $\mathrm{k}$ is the total number of superpixels to be extracted from an image/frame, the total number of pixels in the image/frame is $n$, the pixel value of $i^{\text {th }}$ pixel is represented by $P_{i}$. Fix a 
minimum threshold $T_{1}=0$ and a maximum threshold $T_{2}= \pm 1$ for each superpixel. The pixel value of first pixel in the superpixel is taken as the initial value for that superpixel and the difference between each pixel values are compared with the first pixel value. The equation to determine the difference is,

$$
P(K)=\sum_{i=0}^{n} P_{i-} P_{1}
$$

If the difference of pixel values lies between the threshold $\mathrm{T}_{1}$ (zero) to $T_{2}$ ( \pm one) then the pixel is considered to be in the same superpixel else the pixel belongs to new superpixel.

$$
\mathrm{P}_{\mathrm{i}}=\left\{\begin{array}{r}
\text { if } \mathrm{T}_{1} \leq \mathrm{P}_{\mathrm{i}} \leq \mathrm{T}_{2} \text { then current superpixel } \\
\text { else neighboring superpixel }
\end{array}\right.
$$

Every pixel in the image/frame is undergone to the above mentioned analysis and hence superpixels are extracted from the image/frame. The direction of the pixel which does not lies between the threshold is noted down with respect to first pixel to form a new superpixel in that direction. For example, a pixel below the first pixel is out of the threshold value hence a new superpixel is created in the south of current superpixel and those pixel coordinates that is greater than this pixel alone are taken for comparison. This idea helps in reducing the computation of superpixel extraction which avoids overlapping of superpixels also. The same procedure is followed till it reaches $P_{n}$. This method of superpixel extraction leads to every superpixel approximately with same size and shape. One of the major advantage of this method is every pixels exists within the same superpixel have high correlation and every super pixels have considerably less correlation which will be helpful to easily identify the edges of the object in the image/frame for background subtraction. The space needed to store the pixel values of a image/frame is reduced from thousands to few hundreds. Every superpixel represented in the memory is the representation of group of pixels under the corresponding superpixel.

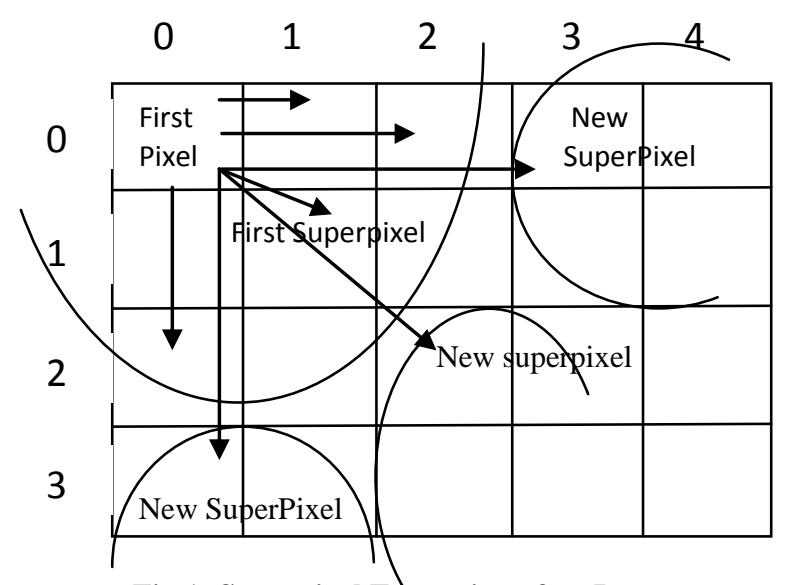

Fig 1: Superpixel Extraction of an Image
The superpixel extraction algorithm is as follows

Input: Image/frame of a video

Output: Image/frame with extraction of superpixels

Method:

1. Initialize the first pixel value $P_{1}$ as initial value of the super pixel

2. Get a pixel Pi

3. Compare $\mathrm{Pi}$ with $\mathrm{P}_{1}$ i.e compute

$$
\mathrm{P}_{\mathrm{i}}-\mathrm{P}_{1}
$$

4. If $\mathrm{Pi}$ lies between $\mathrm{T}_{1}$ and $\mathrm{T}_{2}$ then current superpixel, increment $i$ then goto 2 else goto 5

5. Create next superpixel along the direction of $\mathrm{Pi}$ from current superpixel,

a) Fix the threshold values

b) Initialize the current pixel value as initial pixel value of this superpixel

c) goto 3

6. Stop if $\mathrm{P}_{\mathrm{i}}=\mathrm{P}_{\mathrm{n}}$

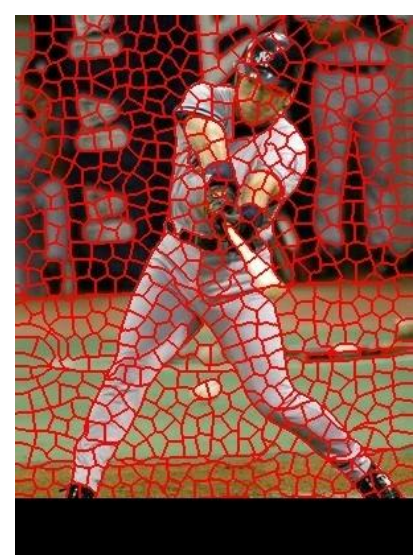

Fig 2: Image after Superpixel Extraction

\section{CANNY EDGE DETECTION}

Canny edge detection algorithm developed by John F.Canny [7] is the most widely used standard algorithm for detecting the edges of an image/frame for past few years due to its optimal characteristics such as low error rate, well localized edge points, response to single edge etc [6] [8]. It is used for feature detection, feature extraction and to capture the changes in image/every frame of a video in background subtraction. The goal of edge detection is to eliminate the irrelevant data to be processed. This algorithm works in five steps: (1) smoothing, (2) finding gradients, (3) non-maximum suppression, (4) color thresholding, and (5) edge tracking by hyterisis [7]. In this paper, the canny edge detection algorithm is modified accordingly to process the superpixels for background subtraction.

First step is to apply the smoothening step which reduces noise from the image/frame using Gaussian filter [7]. Second 
step is to find the gradients of the image. The gradients are obtained for every superpixels but not the pixels of the image. The gradient of the image is determined using Sobel-operator as suggested by Canny [7] which uses Euclidean distance to identify the magnitude. Applying gradient for superpixels reduces the computation complexity in a huge manner that is in terms of number of pixels and parameters undergone for gradient measure. After gradient of superpixel is determined the resulting image/frame shows the marked edges along the superpixel boundaries which may not be the actual object edge because a superpixel is a set of subpixels with similar properties. Thus the obtained image will be of blurred edge (not sharp) due to large superpixel size. The sharp edges are obtained in the next step of Canny edge detection algorithm called non-maximum suppression.

Third step is non-maximum suppression in which the sharp edges are identified from blurred edges [7]. Every subpixel of every superpixel in blurred edges is undergone for nonmaximum suppression such that edge strength of every subpixel is compared with the edge strength of pixels in the positive and negative gradients. Those pixels that have high local maxima and edge strength in this comparison are taken as edge pixels and others are deleted or suppressed. The output image will be marked with sharp edges but the weak edges are still not yet identified accurately due to noise or some rough surfaces. This objective is achieved in the next step called double thresholding.

Fourth step in edge detection is double thresholding to identify the weak edges of the image/frame using three threshold ranges. . Remaining subpixels in every superpixels after non-maximum suppression is undergone for color thresholding. Canny used double thresholding for grayscale images whereas for color images multiband thresholding should be used. For grayscale images two thresholds has to be set, high threshold and low threshold [7]. For color image/frame, it must be converted from RGB image to HSV and three threshold values are used as discussed by MingHsuan Yang and Narendra Ahuja [10]. The important issue to be considered in color thresholding is selection of threshold ranges because the accuracy of output image/frame is also based on these threshold values. Those subpixels that exits within the threshold value are taken as weak pixels, those lie beyond the threshold are strong pixels and those below the threshold value are suppressed. After identifying the weak edges its time to analyze whether the selected weak edges belongs to the edges of the object or noisy pixels. This job is done by the next step of the Canny edge detection called as edge tracking by hyteresis.

Finally edge tracking decides whether a weak subpixel must be included in the edge of the object or not because weak pixels may exist due to noise or color variations in the image/frame [7]. Every weak subpixel is taken as edge pixel only if there is atleast one strong subpixel exists at the 8 connected neighborhood of this subpixel. After edge tracking the complete edges of the object in image/frame is determined effectively using Canny edge detection algorithm with some specified changes that leads to less computation and storage.

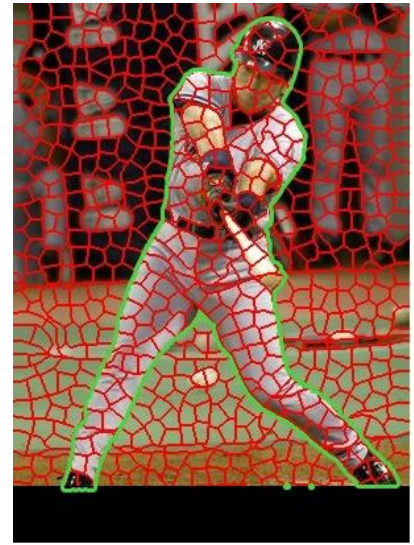

Fig 3: Image after edge detection

\section{FUZZY C MEANS}

Fuzzy c means is most efficient clustering algorithm developed by Dunn [12] and it was improved by Bezdek [13] for segmenting the image which allows a particular pixel to exist in more than one cluster and its degree of membership in those clusters can be identified [10]. This helps to segment the objects of an image efficiently and accurately [11]. Fuzzy c means algorithm is used in this paper to segment the background and foreground object to perform background subtraction of consecutive frames of a video. This algorithm helps to segment the pixels whether it belongs to background of the frame or not. The main advantage of this method is, identifying the pixel membership in each cluster helps to segment the overlapped objects accurately.

The number of clusters is decided based on the objects present in the frames of video used for background subtraction. One of the most important criteria to be considered in fuzzy $\mathrm{c}$ means is choosing the centroid. Initially the centroid is chosen based on the mean of the data points or randomly and the exact centroid can to calculated finally after several iterations of finding the membership degree of pixels. In this paper a new idea is proposed for choosing the centroid based on the superpixels and edge detected using the Canny edge detection. Consider all the superpixels at the left vertical axis of the image/frame, scan every superpixel along horizontal direction till it intersects with superpixel that belongs to the edge of the object marked during the edge detection and let us name it as edge superpixels. The point to be noted is, the strong edge superpixels alone must be taken into consideration for centroid because weak edges may be inner part of the object so during segmentation the entire foreground object is segmented on the whole. Store this edge superpixel and continue scanning horizontally till another edge superpixel is found which means the first edge superpixel and second edge superpixel gives us the boundary of first object in the image/frame. Similarly continue scanning horizontally and note down the third and fourth edge superpixels that represents the boundary of second object in the image/frame.this process continues till the last superpixel in the right vertical axis of the image/frame. If no edge superpixel is found then those superpixels are considered as background superpixels. At the corners of some objects there may not be pair of edge superpixels found hence those edge superpixels without pair belongs to the superpixel of foreground objects.

Now calculate the midpoint between first and second edge superpixel pair. Select any one pixel inside first edge 
superpixel and second edge superpixel along same row and find the midpoint between them by the formula,

$$
\operatorname{Midpoint}_{(1,2)}=\frac{\left(x_{1}, y_{1}\right)+\left(x_{2}, y_{2}\right)}{2}
$$

where $\left(\mathrm{x}_{1}, \mathrm{y}_{1}\right)$ is the coordinate of a pixel in first edge superpixel and $\left(\mathrm{x}_{2}, \mathrm{y}_{2}\right)$ is the coordinate of a pixel in second edge superpixel. Similarly apply the formula for all edge superpixel pairs to identify their midpoints and mark all those midpoints. Join all those midpoints column wise and find the column which contains more number of midpoints in each object. Mark the coordinates of first and last midpoint pixel in that column and find the midpoint for this column using the above formula. The midpoint obtained from this column acts as the centroid for the object.

Apply the fuzzy c means algorithm and segment the objects in the image. The edge pixels already found using Canny edge detection is also used during segmentation to accurately segment the pixels along the boundaries of clusters.

To perform the background subtraction, apply the above mentioned procedure to every frame of a video for segmentation, then find the difference between the previous frame (PF) and current frame $(\mathrm{CF})$ by subtracting the changes in the pixel positions among both. The change detection of a pixel coordinate $(\mathrm{x}, \mathrm{y})$ is obtained from the formula,

$$
\mathrm{CD}(\mathrm{x}, \mathrm{y})=\mathrm{PF}-\mathrm{CF}
$$

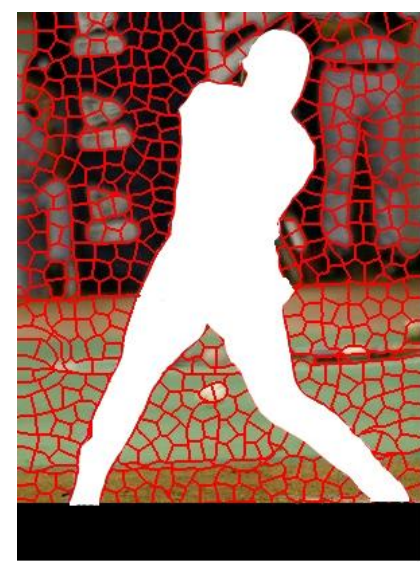

Fig 4: Image after segmentation

\section{RESULTS AND DISCUSSIONS}

The images shown in fig 1-4 are for understanding purpose of readers. After implementation of above mentioned methods, we found a better improvement in accuracy compared to the output obtained from SILK algorithm, also as mentioned above the need of memory is less due to superpixels.

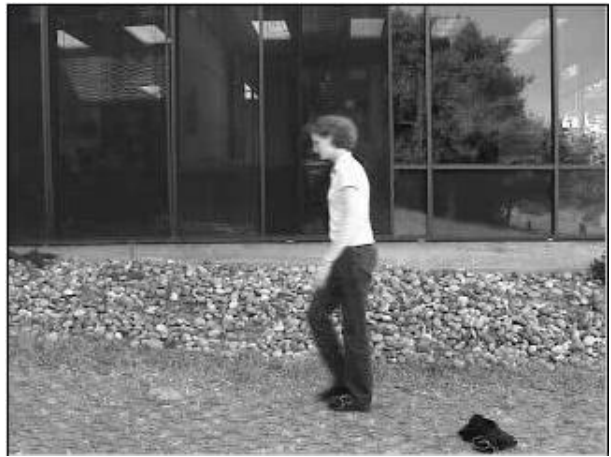

(a)

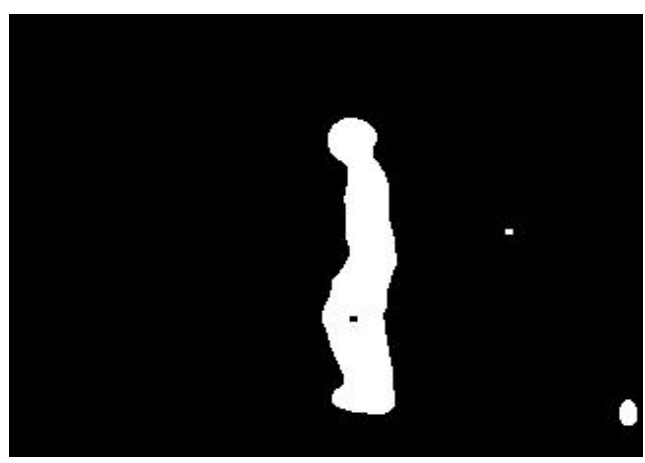

(b)

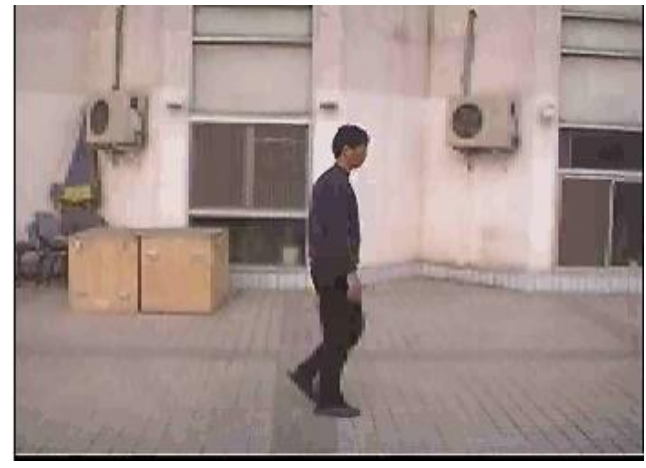

(c)

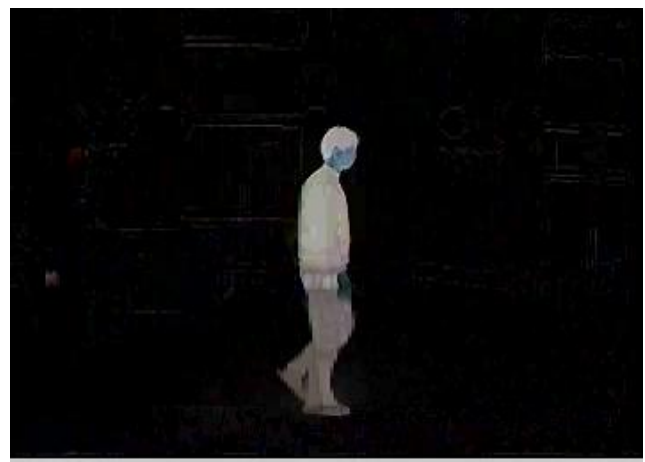

(d)

Fig 5: Background Subtraction b) Using SILK c)Superpixel extraction, Canny edge detection and Fuzzy c means 
The output of SILK algorithm and our method is given in fig 5 , Frame number 26 of a video is shown in fig 5a) and its SILK segmentation in fig $5 \mathrm{~b}$ ). Frame number 28 from another video is taken for implementation of our paper, see fig $5 \mathrm{c}$ ) and its segmentation in $5 \mathrm{~d}$ ).

\section{CONCLUSION}

The superpixel extraction method reduces the processing of thousands of pixels into few hundreds of pixels during the edge detection, segmentation and background subtraction techniques. For background subtraction also superpixels may be taken for finding the difference between current frame and previous frame. After finding the change detection, only those superpixels which are found to be changed can be further reduced to identify the changes in its subpixels. So the processing on superpixels improves the computational complexity in a large extent. Also the space needed to store the superpixel values alone is very less such that $25 \%$ of storage only when compared to the total size of the image/frame. Those superpixels which needs further processing can only be reduced to its subpixels during processing.

The Canny edge detection algorithm is the standard algorithm proved to be most optimal compare to the other edge detection algorithms. By using this edge detection with fuzzy c means segmentation, both edge detection and segmentation of background object and foreground object can be accurately performed so that background subtraction is done efficiently with elimination of noise and sharp edges. Thus the output frames will have accurate marked edges in it which is very useful for real time applications like video surveillance, medical imaging etc.

\section{ACKNOWLEDGMENTS}

The third author is thankful to his Holiness Arulthiru Bangaru Adigalar and her mother Mrs.S.Rajammal for their constant encouragement and motivation. She also wishes to thank her friends Mr.S.Manigandan, Mr.S.Vignesh and Mr.A.Raja for their timely help and support.

\section{REFERENCES}

[1] X. Ren and J. Malik. Learning a classification model for segmentation. In Proc. 9th Int. Conf. Computer Vision, volume 1, pages 10-17, 2003. J. Clerk Maxwell, A Treatise on Electricity and Magnetism, 3rd ed., vol. 2. Oxford: Clarendon, 1892, pp.68-73.

[2] G. Mori, X. Ren, A. Efros, and J. Malik, Recovering Human Body Configurations: Combining Segmentation and Recognition, IEEE Computer Vision and Pattern Recognition, 2004.

[3] G. Mori, Guiding Model Search Using Segmentation, IEEE International Conference on Computer Vision, 2005 .

[4] Cheung, S.-C. and C. Kamath, "Robust Background Subtraction with Foreground Validation for Urban Traffic Video," EURASIP Journal on Applied Signal Processing, Volume 14, pp 1-11, 2005. UCRL-JRNL201916.

[5] Li Cheng, Minglun Gong, Dale Schuurmans and Terry Caelli, "Real Time Discriminative Background Subtraction", in IEEE Transactions on Image Processing, Vol 20, No.5, pages 1401-1414, May 2011.

[6] Sergei Azernikov. Sweeping solids on manifolds. In Symposium on Solid and Physical Modeling, pages 249-255, 2008.
[7] John Canny. A computational approach to edge detection. Pattern Analysis and Machine Intelligence, IEEE Transactions on, PAMI-8(6):679-698, Nov. 1986.

[8] F. Mai, Y. Hung, H. Zhong, and W. Sze. A hierarchical approach for fast and robust ellipse extraction. Pattern Recognition, 41(8):2512-2524, August 2008.

[9] Thomas B. Moeslund. Image and Video Processing. August 2008 .

[10] C. Bezdek, Pattern Recognition with Fuzzy Objective Function Algorithms. New York: Plenum Press, 1981.

[11] J.K. Udupa and S. Samarasekera, "Fuzzy connectedness and object definition: Theory, algorithm and applications in image segmentation," Graphical Models Image Processing, vol.58, no.3, pp.246-261, 1996.

[12] J. C. Dunn (1973): "A Fuzzy Relative of the ISODATA Process and Its Use in Detecting Compact WellSeparated Clusters", Journal of Cybernetics 3: 32-57.

[13] J. C. Bezdek (1981): "Pattern Recognition with Fuzzy Objective Function Algoritms", Plenum Press, New York.

[14] C. Stauffer and W. E. L. Grimson, "Adaptive background mixture models for real-time tracking", IEEE Computer Society Conference on Computer Vision and Pattern Recognition, 1999, 2: 252, 1999.

[15] Z. Zivkovic, "Improved adaptive gaussian mixture model for background subtraction", in Proceedings of the 17th International Conference on Pattern Recognition, 2004. ICPR 2004., volume 2, pages 28-31 Vol.2, 2004.

[16] P. Kaewtrakulpong and R. Bowden, "An improved adaptive background mixture model for realtime tracking with shadow detection", in Proceeding 2nd European Workshop on Advanced Video Based Surveillance Systems, Computer Vision and Distributed Processing, 2001.

[17] O. Javed, K. Shafique, and M. Shah, "A hierarchical approach to robust background subtraction using color and gradient information", in Workshop on Motion and Video Computing, 2002, pages 22-27, 2002.

[18] Hong-xun Zhang and De Xu, "Fusing color and gradient features for background model", in 8th International Conference on Signal Processing, 2006.

[19] Li Cheng, Minglun Gong, Dale Schuurmans and Terry Caelli, "Real Time Discriminative Background Subtraction", in IEEE Transactions on Image Processing, Vol 20, No.5, pages 1401-1414, May 2011.

[20] Y. Ivanov, A. Bobick, and J. Liu, "Fast lighting independent background subtraction", in IEEE Workshop on Visual Surveillance, pages 49-55, 1998.

[21] Ser-nam Lim, Anurag Mittal, Larry S. Davis, and Nikos Paragios, "Fast illumination-invariant background subtraction using two views: Error analysis, sensor placement and applications", IEEE Conference on Computer Vision and Pattern Recognition (CVPR'05), 1: 1071-1078, 2005

[22] Hanzi Wang and D. Suter, "A re-evaluation of mixture of Gaussian background modeling [video signal processing applications]", in IEEE International Conference on Acoustics, Speech, and Signal Processing(ICASSP '05), 2005, volume 2, pages 1017-1020, 2005.

[23] M. Piccardi, "Background subtraction techniques: a review", in IEEE International Conference on Systems, Man and Cybernetics, 2004, volume 4, pages3099-3104,2005. 